\title{
Anomalous origin of the left coronary artery from the pulmonary artery (ALCAPA) in a newborn
}

\author{
Chantale Lapierre • Nicolas Hugues
}

Received: 13 October 2009 /Revised: 18 November 2009/Accepted: 23 December 2009 / Published online: 24 February 2010

(C) Springer-Verlag 2010

A 5-day-old child was referred to the department of cardiology for bradycardia without any other cardiac symptoms. ECG was normal. Cardiac echography demonstrated a reverse flow inside the interventricular branch of the left coronary artery suggesting ALCAPA. ECG-gated multidetector CT angiography, with oblique axial view (a) and 3-D volume-rendered images (b), confirmed the diagnosis of ALCAPA (DLP=99 mGy-cm) (Fig. 1). Reimplantation of the left coronary artery into the aorta was performed promptly and with success.

ALCAPA is a rare congenital anomaly but is one of the most common causes of myocardial ischemia in children [1]. Usually, symptoms occur around 8 weeks of life. If untreated, most patients will die within the first year of life. Cardiac echography alone permits the diagnosis of ALCAPA. Nevertheless, ECG-gated multidetector CT angiography can be used instead of conventional angiography to clarify the anatomy or confirm an unclear diagnosis [2]. In neonates, early surgical correction allows improvement of ventricular function.

C. Lapierre $(\bowtie)$

Department of Medical Imaging,

Sainte-Justine Mother and Child University Hospital Center,

3175 Cote Ste-Catherine Road,

Montreal H3T 1C5, Canada

e-mail: chantal lapierre@ssss.gouv.qc.ca

e-mail: lapierrechantale@hotmail.com

\section{N. Hugues}

Department of Pediatrics, Division of Cardiology, Sainte-Justine Mother and Child University Hospital Center,

Montreal, Canada
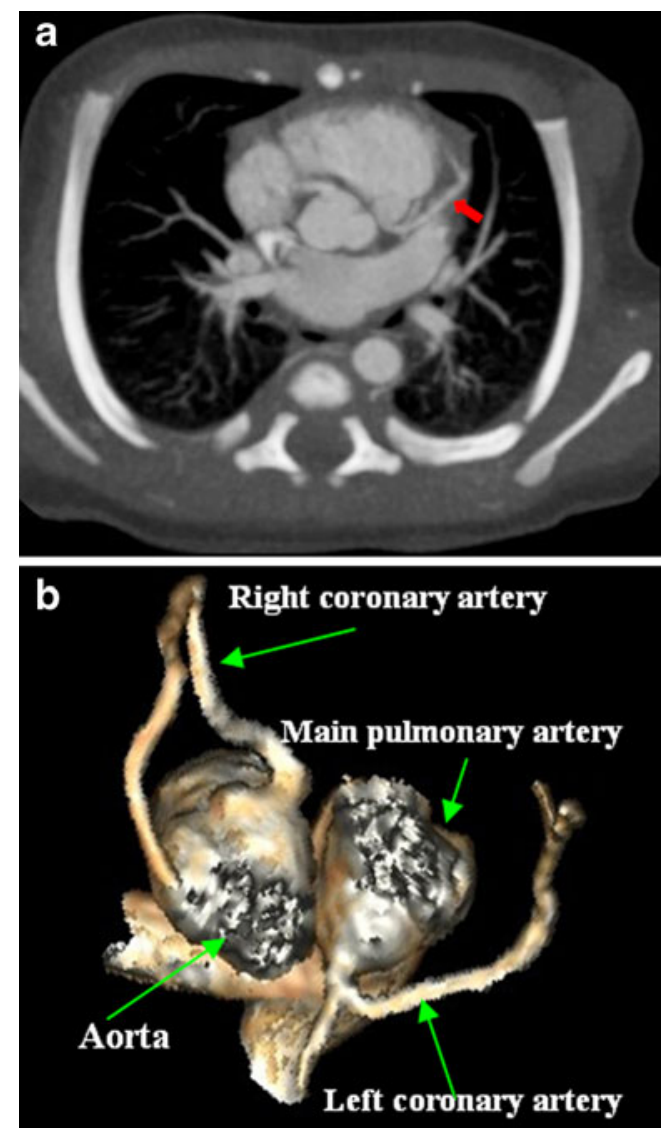

Fig. 1 CT images

\section{References}

1. Lee AC, Foster E, Yeghiazarians Y (2006) Anomalous origin of the left coronary artery from the pulmonary artery: a case series and brief review. Congenit Heart Dis 1:111-115

2. Peña E, Nguyen ET, Merchant N et al (2009) ALCAPA syndrome: not just a pediatric disease. Radiographics 29:553-565 